\title{
Can lycopene be considered an effective protection against cardiovascular disease?
}

\author{
J. Costa-Rodrigues ${ }^{\mathrm{a}, \mathrm{b}, \mathrm{c}, \mathrm{d}, *}$, O. Pinho ${ }^{\mathrm{a}, \mathrm{e}}$, P.R.R. Monteiro ${ }^{\mathrm{b}}$ \\ ${ }^{\text {a }}$ Faculdade de Ciências da Nutrição e Alimentação, U. Porto, Portugal \\ ${ }^{\mathrm{b}}$ ESS - Escola Superior de Saúde, P. Porto, Portugal \\ ${ }^{\mathrm{c}}$ Faculdade de Medicina Dentária, U. Porto, Portugal \\ ${ }^{\mathrm{d}}$ Instituto Politécnico de Viana do Castelo, Escola Superior de Saúde, Portugal \\ ${ }^{\mathrm{e}}$ REQUIMTE/LAQV - U. Porto, Portugal
}

\begin{abstract}
A B S T R A C T
Lycopene is a bioactive component mainly found in tomato. It is characterized by a high antioxidant potential, the highest among carotenoids. Mainly due to this property, lycopene has been suggested to display many beneficial effects, including its potential cardioprotective role. Despite some contradictory observations, which appear to be mainly caused by discrepancies in the different experimental protocols applied in the different studies, growing evidence points to clear benefits of lycopene in the maintenance of cardiovascular function and health. The knowledge about lycopene's preventive effects in atherosclerosis, and other cardiovascular diseases, must be translated into changes in food patterns, aiming to increase the consumption of tomato, tomato-containing products, or other foods with high lycopene content, which can have an important impact on cardiovascular disease, particularly in countries where this represents a major public health concern.
\end{abstract}

Keywords: Lycopene; Cardiovascular diseases; Antioxidant; Anti-inflammatory

\section{Introduction}

Unhealthy food ingestion is a major cause of numerous pathologies, among which are cardiovascular diseases. This group of diseases, which represent the leading cause of death and incapacity worldwide are mainly caused by dysregulated plasma lipid levels, pro-inflammatory status and excessive production of reactive oxygen species (Gonzalez \& Selwyn, 2003). In this context, there are many molecules, some of them not required for human biochemistry and metabolism, which may confer cardioprotection and lower the risk of cardiovascular disease. Among them, antioxidants appear to have a high potential and have been implicated in such protection (Petyaev, 2016).

Lycopene belongs to the family of the lipid-soluble antioxidants called carotenoids, which are present in fruits and vegetables (ElAgamey et al., 2004; Tapiero, Townsend, \& Tew, 2004). Although lycopene can be found in many other fruits and vegetables, it is mainly found in tomatoes, or tomato-containing products, which account for about $80 \%$ of total lycopene ingestion (Bohm, 2012; Moran, Erdman, \& Clinton, 2013). Besides being the carotenoid with the highest antioxidant potential, lycopene has also the ability to modulate several key events that are important in the context of cardiovascular diseases, such as inflammation, apoptosis or cellular communication (Thies, Mills, Moir, \& Masson, 2017). The main disadvantage of lycopene is related to its low bioavailability, which depends not only on the different lycopene biochemical isoforms, but also on the context of ingestion and on the genetics of each individual (Borel, Desmarchelier, Nowicki, \& Bott, 2015; Petyaev, 2016; Zubair et al., 2015). The sum of these variables may account for some of the contradictory information regarding the real effects of lycopene in cardiovascular disease protection. Nevertheless, growing evidence points to robust benefits of lycopene intake, which will be discussed in the present review.

\section{Biochemical properties of lycopene}

Carotenoids are coloured lipid molecules mainly found in vegetable products, such as fruits, leaves, tubers or roots (El-Agamey et al., 2004; Tapiero et al., 2004). They can be divided into three groups, namely, carotenes, xanthophylls and lycopene (Jomova \& Valko, 2013; Rutz, Borges, Zambiazi, da Rosa, \& da Silva, 2016). Globally they present antioxidant properties, and although only a few are indispensable for humans, they are considered very important dietary bioactive components. There are more than 700 different carotenoids found in nature, but only six of them are found in significant amounts in human serum, namely, $\alpha$-carotene, $\beta$-carotene (Fig. 1), $\beta$-cryptoxanthin, zeaxanthin, lutein and lycopene (Maiani et al., 2009). Biochemically they are isoprenoid compounds with a tetraterpene structure (Kaulmann \& Bohn, 
<smiles>CC(=O)C1CCC(C)C(/C=C/C=C(C)/C=C/C=C(C)/C=C/C=C(C)/C=C/C=C(C)/C=C/C2C(C)CCCC2(C)C)C1(C)C</smiles>
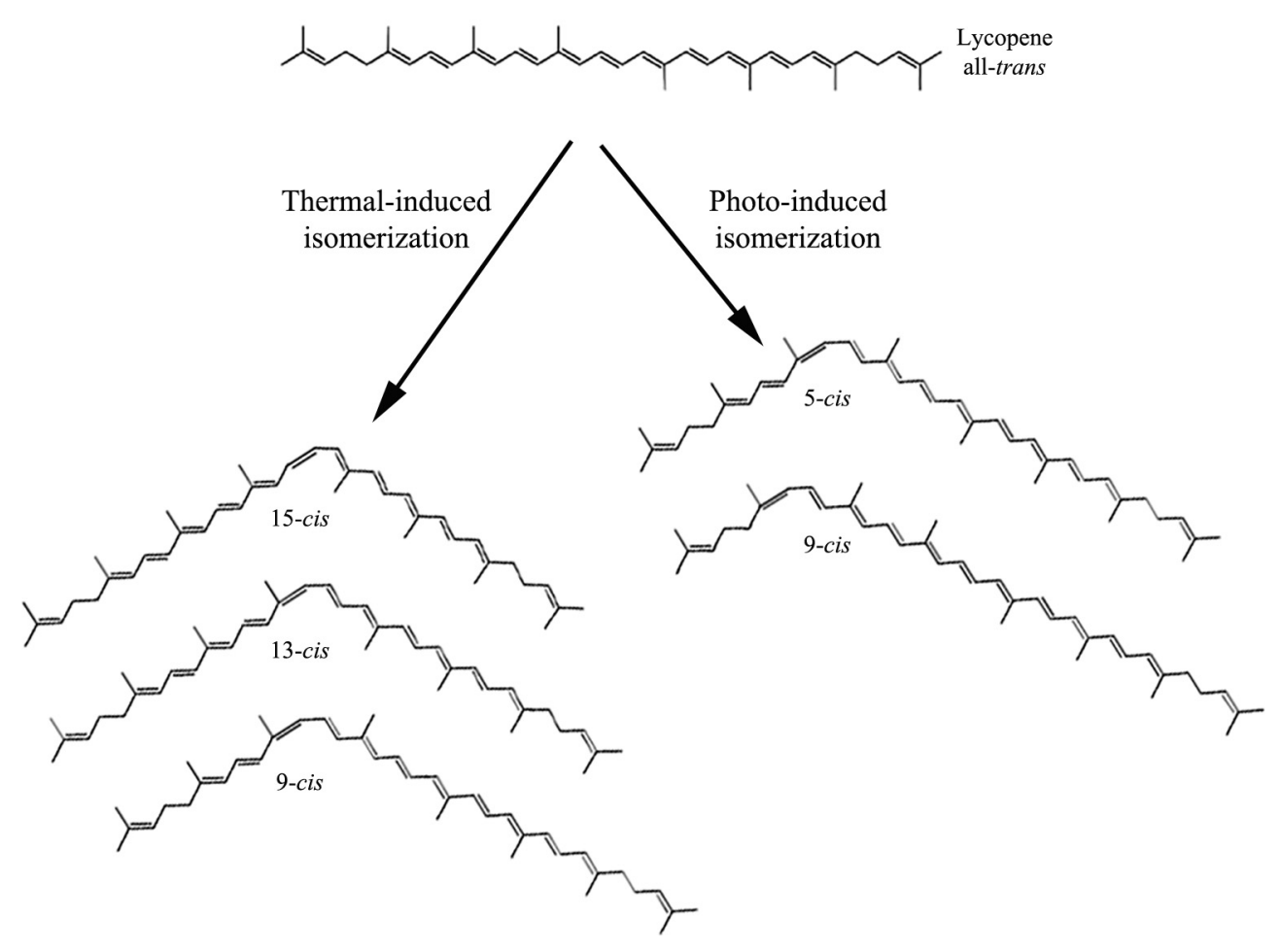

Fig. 1. Chemical structure of $\beta$-carotene, lycopene and its isomers obtained by thermal processing and light exposure.
2014). The majority present a central carbon chain with conjugated double bonds and various cyclic or acyclic groups (Stahl \& Sies, 2005).

Although it was originally isolated from black bryony, lycopene is particularly abundant in tomato (Milani, Basirnejad, Shahbazi, \& Bolhassani, 2016), but it is also found in several other food resources, such as pepper, papaya, watermelon, carrots, pink guava and grapefruit (Assis et al., 2017; Milani et al., 2016). It is a lipophilic molecule with 13 double bonds, which include 11 that are conjugated (Fig. 1) (Petyaev, 2016). In nature, all the double bonds are found in the trans configuration (Jackson, Braun, \& Ernst, 2008). Exposure to light, digestion and thermal processing of lycopene promotes trans-to-cis isomerization (Fig. 1) (Moise, Al-Babili, \& Wurtzel, 2014). This transition is believed to be important to increase the efficiency of its intestinal absorption, a process thought to be mediated by SR-BI but not NPC1L1 membrane transporter (Moussa et al., 2008; Schweiggert et al., 2014). Lycopene's open-polyene chain, without the ionone ring found in $\beta$ carotene, confers this molecule with a very high antioxidant capacity, which is significantly higher than that of other carotenoids, namely, more than twice that of $\beta$-carotene and about 10 times higher than that of $\alpha$-tocopherol (Engelmann, Clinton, \& Erdman, 2011; Milani et al., 2016). It is particularly effective in the quenching of superoxide anion free radicals, although the cis-isoforms are also very effective against peroxyl radicals (Engelmann et al., 2011; Jackson et al., 2008). Although the antioxidant properties of lycopene are well-known, the exact mechanism of action remains to be elucidated (Zhang, Liu, \& Lv, 2016). Serum levels are strongly dependent on the intake of tomato products (Allen et al., 2003; Ganji \& Kafai, 2005), and it is the most abundant carotenoid found in serum in Americans and the second in Europeans (Erdman, Ford, \& Lindshield, 2009; Jenab et al., 2005).

Its half-life in humans also depends on the configuration of the conjugated double bonds, being 5.3 and 8.8 days for all-trans and all-cis isomers, respectively (Moran et al., 2015). One of the main obstacles, when considering the biological effects of lycopene, is its very low and variable bioavailability, which depends on the combination of at least twenty-eight single nucleotide polymorphisms (Borel et al., 2015; Zubair et al., 2015). Other factors accounting for this situation include the configuration of the double bonds, the geographic location of individuals, other sociodemographic factors, health status and, obviously, the food ingestion context (Petyaev, 2016). This high complexity may account, at least partially, for the elevated inter-individual variability of lycopene concentrations and biological effects. Moreover, lycopene distribution throughout the human body is asymmetrical, being mostly found in liver, adrenals, lungs, prostate and skin (Moran, Clinton, \& Erdman, 2013).

Plasma levels of lycopene are very different among different populations and may reflect not only the endogenous properties of each individual, but, as previously mentioned, the diet. Globally, Northern and Western Europe along with America seem to be the regions with the lower ingestion of lycopene, while Asia and Central Africa appears as the region with the highest values (Murphy, Barraj, Spungen, Herman, \& Randolph, 2014). The main dietary source of lycopene, worldwide, is tomato (a significantly higher intake than carrots); however, in Asia, lycopene comes mainly from watermelon $(\sim 50 \%)$, but also from tomatoes $(\sim 30 \%)$. Regarding the high plasma levels of lycopene found in Asia, it is important to highlight that that region is becoming one of the largest worldwide tomato producers, which may account for the observed plasma levels. Furthermore, it is estimated that tomato consumption accounts for $10-20 \%$ of the total vegetal consumption in the Pacific region of Asia. Also, metabolic relationships on the bioavailability and utilization of lycopene, as well as lifestyle, may contribute to the observations highlighted before, significantly accounting for the differences observed in lycopene plasma values among the world.

Lycopene has the potential to modulate many different cellular processes. There are multiple forms by which it can act in target cells 
(Feitelson et al., 2015; Friedman, 2013; Thies et al., 2017). It was observed that lycopene can promote apoptosis and inhibit cell proliferation both in vitro and in vivo (Gupta, Bansal, \& Koul, 2013; Uppala, Dissmore, Lau, Andacht, \& Rajaram, 2013). Also, it is able to stimulate cell differentiation in vitro, particularly due to a positive effect in gap junction communication (Stahl, von Laar, Martin, Emmerich, \& Sies, 2000). Other important mechanisms of action of lycopene include the inhibition of angiogenesis and oxidative damage, and the stimulation of phase II enzymes expression and activity, both in vitro and in vivo (Chen, Lin, Yang, \& Hu, 2012; Lian \& Wang, 2008; Palozza, Simone, Catalano, Russo, \& Bohm, 2012). Also, as discussed below, lycopene have the ability to modulate inflammation, which may account for its health potential benefits.

Since oxidative stress has been linked to numerous pathological conditions, carotenoid ingestion, and, particularly, lycopene consumption, is associated to a decrease in the risk of chronic conditions such as inflammatory disorders, cancer, gastrointestinal, cardiovascular and neurodegenerative diseases, as well as to an increase in the immune system function (Ghavipour et al., 2013; Lorenz et al., 2012; Milani et al., 2016). Nevertheless, as highlighted in the present review, the mechanisms of action of lycopene lay far beyond its antioxidant properties and, thus, the potential effects of lycopene on human cells are multiple.

\section{Pathophysiology of cardiovascular disease}

Cardiovascular diseases are the main cause of mortality and morbidity around the world. They present a complex pathogenesis, the majority of them being caused by ischemic events and, globally, they can be considered as the result of a sum of different complementary factors, such as increased blood lipid levels and blood pressure, as well as endothelial dysfunction (Gonzalez \& Selwyn, 2003). Although nowadays there are numerous technical interventions that can be conducted in order to treat patients, such as coronary bypass surgery, thrombolysis, or angioplasty (Tong et al., 2016), special attention must be taken regarding primary prevention, since this group of diseases is mainly caused by modifiable risk factors. Among them, perhaps the most important one is diet. It is well-known that Mediterranean countries have a lower rate of cardiovascular disease mortality (GarciaFernandez, Rico-Cabanas, Rosgaard, Estruch, \& Bach-Faig, 2014), which is at least partially attributable to the healthy eating lifestyle. The so-called Mediterranean diet is based on the ingestion of high amounts of fresh fruit and vegetables, with one of the most ingested being the tomato (Petyaev, 2016).

In the context of cardiovascular ischemic diseases, it is also important to considerer that reperfusion after prolonged ischemia may lead to myocardial injury, which, in turn, can cause infarct expansion and/or arrhythmias (Tong et al., 2016). However, either in the case of the initial lesion, or in the case of reperfusion lesions, oxidative stress appears to have a central role (Tong et al., 2016). Thus, high antioxidant levels, including those of lycopene, may contribute in preventing or, at least, decreasing, the extent of these lesions.

Cardiovascular diseases have a complex pathophysiology, with several parameters critical for their development and, consequently, for the clinical outcome. The main parameters are summarized in Table 1.
One key event in the development of the vast majority of cardiovascular diseases is the oxidation of low density lipoprotein (LDL) (Aviram, 1993; Steinberg \& Witztum, 2010). Its accumulation in the sub-endothelial space, and further recognition by the scavenger receptors found on the surface of macrophages, causes the formation of foam cells, which are characteristic of the atherosclerotic lesions. Besides this important role in the pathogenesis of atherosclerosis, oxidized LDL also promotes endothelial dysfunction, increases the production of superoxide anion radical and the remodeling of the smooth muscle layers on vascular walls (Galle, Hansen-Hagge, Wanner, \& Seibold, 2006). In this context, the high antioxidant potential of lycopene may account for a decrease in LDL oxidation and, thus, decrease the risk of cardiovascular disease. For the other two parameters (blood pressure and flow-mediated dilation), the action of $\mathrm{NO}$ as a key vasodilator appears to be strongly dependent on the redox status of the cellular environment, since the endothelial-derived free radicals may neutralize it. So, lycopene, as well as other antioxidants, may have an important role in the prevention of excessive NO neutralization and, consequently, in the maintenance of a proper vessel diameter. Taken together, there are numerous factors involved in the genesis and development of cardiovascular diseases, and the majority of them seem to have the potential for being modulated by bioactive compounds, including lycopene.

\section{Cardioprotective properties of lycopene}

\subsection{Controversies}

Due to the complex nature of cardiovascular disease, many dietary phytochemical properties have been suggested to play an important role in cardiovascular protection. For example, the consumption of nitrate-rich food, due to its potential to increase NO plasma levels, has been linked to improvements in endothelial integrity and function (Lara et al., 2016) and also to a decrease in blood pressure (Siervo, Lara, Ogbonmwan, \& Mathers, 2013). Also, dietary antioxidants, such as carotenoids, appear to be potential key players in the prevention of cardiac and vessels pathologies (Mozaffarian, Appel, \& Van Horn, 2011). In line with this, in a meta-analysis of sixteen cohort studies, involving more than 800,000 subjects, it was observed that the mortality associated with cardiovascular disease is lowered by a higher consumption of fruit and vegetables, namely, up to five servings/day (Wang et al., 2014). This effect appeared to be sensitive to the number of servings.

Since among carotenoids, lycopene is the one with the highest antioxidant potential, generally it has been linked to a decrease in cardiovascular disease risk (Jacques, Lyass, Massaro, Vasan, \& D'Agostino, 2013; Sesso, Liu, Gaziano, \& Buring, 2003; Yeo, Kim, Lim, Kim, \& Lee, 2011). Although theoretically this relationship is consistent with the knowledge of the impact of oxidative stress in cardiovascular disease, and there is growing evidence that points that way (Table 2), in practice it remains, at least in some aspects, controversial.

Although the majority of the studies are favourable to positive effects of lycopene on cardiovascular health, there are also several studies that have failed to prove a consistent relationship between lycopene intake and a decreased risk of cardiovascular disease markers or outcome (Li \& Xu, 2014; Osganian et al., 2003; Sesso et al., 2003; Tavani,

Table 1

Impact of different cardiovascular parameters on cardiovascular disease.

\begin{tabular}{|c|c|c|}
\hline Cardiovascular parameter & Normal healthy levels & Cardiovascular disease outcome \\
\hline LDL levels ${ }^{\text {a) }}$ & $<2.6 \mathrm{mM}$ & Reduction of $1 \mathrm{mM}$ elicits a decrease of $23 \%$ in the risk of occurrence of myocardial infarction and $12 \%$ in all-cause mortality \\
\hline Blood pressure $\mathrm{b}^{\mathrm{b}}$ & $<120 / 80 \mathrm{mmHg}$ & $\begin{array}{l}\text { Reduction of } 5 \mathrm{mmHg} \text { cause a decline in cardiovascular disease mortality of about } 9 \% \text {, with a decrease of } 13-14 \% \text { in the case } \\
\text { of stroke }\end{array}$ \\
\hline Flow-mediated dilation ${ }^{\mathrm{c})}$ & $7-10 \%$ & Increase of $1 \%$ causes a reduction of $10-13 \%$ in the risk of cardiovascular pathological events \\
\hline
\end{tabular}

a) Baigent et al., 2005/b) Chobanian et al., 2003; Reboldi et al., 2011/c) Matsuzawa, Kwon, Lennon, Lerman, \& Lerman, 2015 ; Xu et al., 2014. 
Table 2

Main cardiovascular protective effects of lycopene.

\begin{tabular}{ll}
\hline Effects & Reference \\
\hline$\downarrow$ ischemic heart disease & - Riccioni et al., 2009 \\
$\downarrow$ coronary insufficiency & - Wolak \& Paran, 2013 \\
$\uparrow$ HDL functionality & - Thies et al., 2012 \\
$\downarrow$ plasma LDL-cholesterol levels & - Cheng et al., 2017 \\
$\uparrow$ endothelial function & \\
$\downarrow$ risk of cardiovascular disease & - Song et al., 2017 \\
$\downarrow$ arterial thickness & - Karppi et al., 2013 \\
$\downarrow$ risk of atherosclerosis and/or stroke & \\
$\uparrow$ protection of endothelial cells from oxidative & - Hung et al., 2008 \\
$\quad$ damage & \\
$\downarrow$ monocyte-endothelium interactions & - Sawardekar et al., 2016 \\
$\downarrow$ platelet aggregation & \\
$\downarrow$ oxidative response of neutrophils & - Marcotorchino et al., 2012 \\
$\downarrow$ secretion of pro-inflammatory cytokines & - Zou et al., 2013 \\
$\downarrow$ proliferation of smooth muscle cells & - Lo et al., 2007 \\
$\downarrow$ activation of monocytes and T-lymphocytes & - Mills et al., 2012 \\
$\downarrow$ C-reactive protein levels in women & - Biddle et al., 2015 \\
$\uparrow$ vasodilation mediated by endothelial cells & - Gajendragadkar et al., 2014 \\
\hline
\end{tabular}

Gallus, Negri, Parpinel, \& La Vecchia, 2006; Thies et al., 2017). These include human intervention studies, prospective studies, and retrospective studies. There might be many different reasons underlying these negative associations. As stated above, lycopene bioavailability and metabolism is strongly influenced by genetic variability, being described at least 28 single nucleotide polymorphisms in 16 genes, among which are those coding for the cholesterol membrane transporter scavenger receptor class B, member 1 (SCARB1), the molecular guidance cue slit homolog 3 gene (SLIT3) and the steroid-breakdown enzyme dehydrogenase/reductase (SDR family) member 2 (DHRS2) (Borel et al., 2015; Zubair et al., 2015) and, thus, results would be significantly influenced by this issue. Also, the cardiovascular markers used in the different studies varied significantly (ranging from biochemical parameters such as LDL levels or blood pressure, to clinical outcomes like occurrence of myocardial infarction, stroke or death) which makes detailed comparisons among them difficult. The variability found in the lycopene sources (ranging from fresh tomatoes to processed tomatoes, tomato-containing foods or even lycopene supplements), lycopene doses, ingestion of foods containing other compounds that may potentiate or decrease lycopene effects and duration of the experiments, may also account for some of the observed discrepancies. Furthermore, many published works used less than 100 volunteers, which decreases their statistical power and, thus, caution is required in the interpretation and extrapolation of the results. Knowing in advance that there are no perfect experimental protocols, it is recommended that future retrospective experiments should be conducted with large populations (preferably from the same geographic location, in order to avoid a high genetic variability), if possible framed in cohorts well-characterized from a food pattern point of view. At the same time, prospective studies, in which the amount and processing of tomatoes ingested are strictly controlled, may be informative at the level of possible biochemical alterations that decrease or increase the risk of developing cardiovascular disease. In this case, special attention must be paid to the experimental period, since lycopene ingested prior to the start of the study may persist in the body for a significant time. Thus, a period of washout of lycopene from previous ingestion is recommended.

\subsection{Lycopene and cardiovascular risk factors}

Recently, the impact of lycopene supplementation in cardiovascular disease risk factors was reviewed (Burton-Freeman \& Sesso, 2014). Once again, the different experimental protocols found in the different studies caused difficulties in the comparison of results, but it appeared that the consumption of lycopene-containing food may be more effective than the use of lycopene supplements. This is probably due to the presence of other important compounds in tomato products, such as bioactive molecules and also electrolytes, that may potentiate the lycopene bioavailability and the lycopene-related effects (Friedman, 2013). In another recent study, an inverse association was observed between lycopene consumption and the incidence of ischemic heart disease, as well as coronary insufficiency, which is in line with the previous findings (Riccioni et al., 2009; Wolak \& Paran, 2013).

In a randomized trial performed with 225 volunteers, the authors were unable to demonstrate significant effects of lycopene consumption, from tomato-containing food, on conventional inflammatory, vascular and lipid markers. However, lycopene elicited an improvement in high density lipoprotein (HDL) functionality (Thies et al., 2012).

\subsection{Lycopene and cardiovascular function}

Recently, a systematic review and meta-analysis was conducted, concerned with the effects of lycopene and cardiovascular function (Cheng et al., 2017). Only interventional studies with human subjects with dietary exposure to tomato-based products or lycopene supplements were considered, giving a total of twenty-two publications. Globally, it was observed that not only did lycopene appear to elicit a decrease in plasma LDL-cholesterol levels, but also an increase in endothelial function. Regarding the former effect, it is important to highlight that once again, only lycopene-containing foods, but not lycopene supplements, were able to promote it. Furthermore, a decrease in blood pressure (both systolic and diastolic), triglycerides, inflammation and adhesion markers, as well as an increase in HDL-cholesterol, also seemed to be linked to a high ingestion of lycopene-containing food/supplements, although in this case, the evidence was not so robust (Cheng et al., 2017). These results are in line with previous meta-analysis studies in this area (Li \& Xu, 2013; Ried \& Fakler, 2011). Interestingly, important differences among different countries were also highlighted, which suggests that the geographic context and culture may also modulate the cardioprotective effects of lycopene (Cheng et al., 2017; Li \& Xu, 2013; Ried \& Fakler, 2011).

In a recent meta-analysis study, aiming to evaluate the relationship between lycopene intake, its plasma levels and cardiovascular disease, it was observed that lycopene was associated with a $17 \%$ reduction in the risk of cardiovascular disease, when comparing the highest analyzed intake with the lowest one (Song et al., 2017), The reduction of cholesterol levels and inflammatory response, the decreased oxidation of biomolecules, the improvement of intercellular communications and stimulation of apoptosis and the antiangiogenic effects were among the proposed mechanisms involved in lycopene's cardiovascular protective effects. The effects on cholesterol levels may be attributable to different actions, like inhibition of cholesterol synthesis and modulation of LDL receptor and intracellular storage of cholesterol (Palozza, Catalano, Simone, Mele, \& Cittadini, 2012).

\subsection{Lycopene and inflammation}

The anti-inflammatory effects of lycopene have been linked to numerous ways in which this molecule can modulate cellular activities. High lycopene levels seemed to be inversely related with arterial thickness and, putatively, with the risk of atherosclerosis and/or stroke (Karppi, Kurl, Ronkainen, Kauhanen, \& Laukkanen, 2013). Also, it can directly protect endothelial cells from oxidative damage, and inhibit monocyte-endothelium interactions, as well as platelet aggregation (Hung et al., 2008; Sawardekar, Patel, \& Uchil, 2016). Furthermore, lycopene may suppress the oxidative response of neutrophils, as well as the secretion of pro-inflammatory cytokines (Marcotorchino et al., 2012; Zou, Feng, Ling, \& Duan, 2013). The proliferation of smooth muscle cells, as well as monocytes and T-lymphocytes, also appear to be downregulated by lycopene (Lo et al., 2007; Mills, Wilson, \& Thies, 2012). 
In a study conducted with heart failure patients, lycopene consumption promoted a decrease in C-reactive protein levels in women, but had no significant effect in men (Biddle et al., 2015). In another study, lycopene supplementation was able to improve vasodilation mediated by endothelial cells, in cardiovascular disease patients, while in the healthy control patients, no significant effect was observed (Gajendragadkar et al., 2014), suggesting that lycopene may be particularly effective as a secondary prevention player.

Thus, although lycopene presents a very high antioxidant activity, at least in some cases its role in cardiovascular protection appears to be more related to its anti-inflammatory properties, than to the inhibition of the oxidation of LDL, since lycopene, due to its elevated hydrophobicity, is more likely to be found in the nuclear hydrophobic core of the lipoprotein (Muller, Caris-Veyrat, Lowe, \& Bohm, 2016). Nevertheless, it was proposed that the consumption of one or more servings/ day of tomato-rich products is related to a lower risk (30\%) of cardiovascular disease (Sesso et al., 2003). Quantitatively, the cardioprotective effects of lycopene seemed to be relevant following 1-180 days of tomato-products ingestion (70-400 g/day) (Cheng et al., 2017). For comparison, the average consumption of lycopene ranges between 5 and $7 \mathrm{mg}$ /day in the developed countries, which corresponds to $\sim 50 \mathrm{~g}$ of tomato-products (Wang, 2012).

It is important to note that the conflicting results about the potential cardioprotection elicited by lycopene may be related to the wide variety of experimental protocols used to discover any association between lycopene consumption and cardiovascular disease. These important differences include the pre-existing levels of lycopene, the source of lycopene (tomato products, supplements, etc.), the characteristics of the target populations (healthy, unhealthy, smokers, non-smokers, etc.), the amount of lycopene consumed, the duration of lycopene supplementation and the analyzed markers/cellular effects. Interestingly, and despite all the observed differences, it was proposed that total carotenoid levels may predict all-cause mortality and poor outcomes and rapid progression of cardiovascular disease (Shardell et al., 2011). Although, globally, carotenoids are not indispensable micronutrients, they have very important biochemical features that make them useful allies in promoting a good health status.

\section{Conclusion}

Despite some controversy about specific effects of lycopene in cardiovascular protection, growing evidence points to unequivocal benefits of lycopene intake both in terms of cardiac, endothelial and vascular function and health. The underlying mechanisms by which lycopene exerts its effects are now being unravelled, and might explain some of the contradictions observed in the literature. The genetic inter-individual differences may also be a key issue in understanding the real effects of lycopene in each individual. The context of ingestion of lycopene also appears to be important, since globally the beneficial effects were more significant for the ingestion of food-containing lycopene (particularly tomato products), than for lycopene supplements. Thus, the ingestion of lycopene-containing food, may be relevant for the prevention of atherosclerosis and other cardiovascular diseases and, consequently, must be considered an important strategy, particularly in countries where these diseases are a major public health concern. Also, conditions that promote long-lasting oxidative stress, such as physical exercise, smoking or chronic pathologies, may cause a significant decrease in lycopene levels, and, thus, an efficient replenishment of that molecule may be required. Taken together, recommendations for lycopene intake must take into account not only its low bioavailability and asymmetrical distribution throughout the human body, but also the conditions that can accelerate the loss of lycopene. So, the establishment of individualized nutritional approaches and recommendations that consider all of these factors may have a profound impact on cardiovascular disease and, consequently, on human health.

\section{References}

Allen, C. M., Schwartz, S. J., Craft, N. E., Giovannucci, E. L., De Groff, V. L., \& Clinton, S. K. (2003). Changes in plasma and oral mucosal lycopene isomer concentrations in healthy adults consuming standard servings of processed tomato products. Nutrition and Cancer, 47, 48-56.

Assis, R. P., Arcaro, C. A., Gutierres, V. O., Oliveira, J. O., Costa, P. I., Baviera, A. M., \& Brunetti, I. L. (2017). Combined effects of curcumin and lycopene or bixin in yoghurt on inhibition of LDL oxidation and increases in HDL and paraoxonase levels in streptozotocin-diabetic rats. International Journal of Molecular Sciences, 18, E332.

Aviram, M. (1993). Modified forms of low density lipoprotein and atherosclerosis. Atherosclerosis, 98, 1-9.

Baigent, C., Keech, A., Kearney, P. M., Blackwell, L., Buck, G., Pollicino, C., et al. (2005) Efficacy and safety of cholesterol-lowering treatment: Prospective meta-analysis of data from 90,056 participants in 14 randomised trials of statins. Lancet, 366, 1267-1278.

Biddle, M. J., Lennie, T. A., Bricker, G. V., Kopec, R. E., Schwartz, S. J., \& Moser, D. K. (2015). Lycopene dietary intervention: A pilot study in patients with heart failure. Journal of Cardiovascular Nursing, 30, 205-212.

Bohm, V. (2012). Lycopene and heart health. Molecular Nutrition \& Food Research, 56, 296-303.

Borel, P., Desmarchelier, C., Nowicki, M., \& Bott, R. (2015). Lycopene bioavailability is associated with a combination of genetic variants. Free Radical Biology \& Medicine, 83 238-244.

Burton-Freeman, B., \& Sesso, H. D. (2014). Whole food versus supplement: Comparing the clinical evidence of tomato intake and lycopene supplementation on cardiovascular risk factors. Advances in Nutrition, 5, 457-485.

Chen, M. L., Lin, Y. H., Yang, C. M., \& Hu, M. L. (2012). Lycopene inhibits angiogenesis both in vitro and in vivo by inhibiting MMP-2/uPA system through VEGFR2-mediated PI3K-Akt and ERK/p38 signaling pathways. Molecular Nutrition \& Food Research, $56,889-899$.

Cheng, H. M., Koutsidis, G., Lodge, J. K., Ashor, A., Siervo, M., \& Lara, J. (2017). Tomato and lycopene supplementation and cardiovascular risk factors: A systematic review and meta-analysis. Atherosclerosis, 257, 100-108.

Chobanian, A. V., Bakris, G. L., Black, H. R., Cushman, W. C., Green, L. A., Izzo, J. L., Jr., et al. (2003). Seventh report of the Joint National Committee on Prevention, Detection, Evaluation, and Treatment of High Blood Pressure. Hypertension, 42, 1206-1252.

El-Agamey, A., Lowe, G. M., McGarvey, D. J., Mortensen, A., Phillip, D. M., Truscott, T. G., et al. (2004). Carotenoid radical chemistry and antioxidant/pro-oxidant properties. Archives of Biochemistry and Biophysics, 430, 37-48.

Engelmann, N. J., Clinton, S. K., \& Erdman, J. W., Jr. (2011). Nutritional aspects of phytoene and phytofluene, carotenoid precursors to lycopene. Advances in Nutrition, 2, 51-61.

Erdman, J. W., Jr., Ford, N. A., \& Lindshield, B. L. (2009). Are the health attributes of lycopene related to its antioxidant function? Archives of Biochemistry and Biophysics, 483, 229-235.

Feitelson, M. A., Arzumanyan, A., Kulathinal, R. J., Blain, S. W., Holcombe, R. F., Mahajna, J., et al. (2015). Sustained proliferation in cancer: Mechanisms and novel therapeutic targets. Seminars in Cancer Biology, 35(Suppl.), S25-S54.

Friedman, M. (2013). Anticarcinogenic, cardioprotective, and other health benefits of tomato compounds lycopene, alpha-tomatine, and tomatidine in pure form and in fresh and processed tomatoes. Journal of Agricultural and Food Chemistry, 61, 9534-9550.

Gajendragadkar, P. R., Hubsch, A., Maki-Petaja, K. M., Serg, M., Wilkinson, I. B., \& Cheriyan, J. (2014). Effects of oral lycopene supplementation on vascular function in patients with cardiovascular disease and healthy volunteers: A randomised controlled trial. PLOS ONE, 9, e99070.

Galle, J., Hansen-Hagge, T., Wanner, C., \& Seibold, S. (2006). Impact of oxidized low density lipoprotein on vascular cells. Atherosclerosis, 185, 219-226.

Ganji, V., \& Kafai, M. R. (2005). Population determinants of serum lycopene concentrations in the United States: Data from the Third National Health and Nutrition Examination Survey, 1988-1994. Journal of Nutrition, 135, 567-572.

Garcia-Fernandez, E., Rico-Cabanas, L., Rosgaard, N., Estruch, R., \& Bach-Faig, A. (2014). Mediterranean diet and cardiodiabesity: A review. Nutrients, 6, 3474-3500.

Ghavipour, M., Saedisomeolia, A., Djalali, M., Sotoudeh, G., Eshraghyan, M. R. Moghadam, A. M., et al. (2013). Tomato juice consumption reduces systemic inflammation in overweight and obese females. British Journal of Nutrition, 109, 2031-2035.

Gonzalez, M. A., \& Selwyn, A. P. (2003). Endothelial function, inflammation, and prognosis in cardiovascular disease. The American Journal of Medicine, 115(Suppl. 8A), 99S-106S.

Gupta, P., Bansal, M. P., \& Koul, A. (2013). Evaluating the effect of lycopene from Lycopersicum esculentum on apoptosis during NDEA induced hepatocarcinogenesis. Biochemical and Biophysical Research Communications, 434, 479-485.

Hung, C. F., Huang, T. F., Chen, B. H., Shieh, J. M., Wu, P. H., \& Wu, W. B. (2008) Lycopene inhibits TNF-alpha-induced endothelial ICAM-1 expression and monocyteendothelial adhesion. European Journal of Pharmacology, 586, 275-282.

Jackson, H., Braun, C. L., \& Ernst, H. (2008). The chemistry of novel xanthophyll carotenoids. American Journal of Cardiology, 101, 50D-57D.

Jacques, P. F., Lyass, A., Massaro, J. M., Vasan, R. S., \& D'Agostino, R. B., Sr. (2013). Relationship of lycopene intake and consumption of tomato products to incident CVD. British Journal of Nutrition, 110, 545-551.

Jenab, M., Ferrari, P., Mazuir, M., Tjonneland, A., Clavel-Chapelon, F., Linseisen, J., et al (2005). Variations in lycopene blood levels and tomato consumption across European 
countries based on the European Prospective Investigation into Cancer and Nutrition (EPIC) study. Journal of Nutrition, 135, 2032S-2036S.

Jomova, K., \& Valko, M. (2013). Health protective effects of carotenoids and their interactions with other biological antioxidants. European Journal of Medicinal Chemistry, 70, 102-110.

Karppi, J., Kurl, S., Ronkainen, K., Kauhanen, J., \& Laukkanen, J. A. (2013). Serum carotenoids reduce progression of early atherosclerosis in the carotid artery wall among Eastern Finnish men. PLoS ONE, 8, e64107.

Kaulmann, A., \& Bohn, T. (2014). Carotenoids, inflammation, and oxidative stress-implications of cellular signaling pathways and relation to chronic disease prevention. Nutrition Research, 34, 907-929.

Lara, J., Ashor, A. W., Oggioni, C., Ahluwalia, A., Mathers, J. C., \& Siervo, M. (2016). Effects of inorganic nitrate and beetroot supplementation on endothelial function: A systematic review and meta-analysis. European Journal of Nutrition, 55, 451-459.

Li, X., \& Xu, J. (2013). Lycopene supplement and blood pressure: An updated metaanalysis of intervention trials. Nutrients, 5, 3696-3712.

Li, X., \& Xu, J. (2014). Dietary and circulating lycopene and stroke risk: A meta-analysis of prospective studies. Scientific Reports, 4, 5031.

Lian, F., \& Wang, X. D. (2008). Enzymatic metabolites of lycopene induce Nrf2-mediated expression of phase II detoxifying/antioxidant enzymes in human bronchial epithelial cells. International Journal of Cancer, 123, 1262-1268.

Lo, H. M., Hung, C. F., Tseng, Y. L., Chen, B. H., Jian, J. S., \& Wu, W. B. (2007). Lycopene binds PDGF-BB and inhibits PDGF-BB-induced intracellular signaling transduction pathway in rat smooth muscle cells. Biochemical Pharmacology, 74, 54-63.

Lorenz, M., Fechner, M., Kalkowski, J., Frohlich, K., Trautmann, A., Bohm, V., et al. (2012). Effects of lycopene on the initial state of atherosclerosis in New Zealand White (NZW) rabbits. PLoS ONE, 7, e30808.

Maiani, G., Caston, M. J., Catasta, G., Toti, E., Cambrodon, I. G., Bysted, A., et al. (2009). Carotenoids: Actual knowledge on food sources, intakes, stability and bioavailability and their protective role in humans. Molecular Nutrition \& Food Research, 53(Suppl. 2), S194-S218.

Marcotorchino, J., Romier, B., Gouranton, E., Riollet, C., Gleize, B., Malezet-Desmoulins, C., et al. (2012). Lycopene attenuates LPS-induced TNF-alpha secretion in macrophages and inflammatory markers in adipocytes exposed to macrophage-conditioned media. Molecular Nutrition \& Food Research, 56, 725-732.

Matsuzawa, Y., Kwon, T. G., Lennon, R. J., Lerman, L. O., \& Lerman, A. (2015). Prognostic value of flow-mediated vasodilation in brachial artery and fingertip artery for cardiovascular events: A systematic review and meta-analysis. Journal of the American Heart Association, 4, e002270.

Milani, A., Basirnejad, M., Shahbazi, S., \& Bolhassani, A. (2016). Carotenoids: Biochemistry, pharmacology and treatment. British Journal of Pharmacology, 174, 1290-1324.

Mills, L. M., Wilson, H., \& Thies, F. (2012). Lycopene inhibits lymphocyte proliferation through mechanisms dependent on early cell activation. Molecular Nutrition \& Food Research, 56, 1034-1042.

Moise, A. R., Al-Babili, S., \& Wurtzel, E. T. (2014). Mechanistic aspects of carotenoid biosynthesis. Chemical Reviews, 114, 164-193.

Moran, N. E., Cichon, M. J., Riedl, K. M., Grainger, E. M., Schwartz, S. J., Novotny, J. A., et al. (2015). Compartmental and noncompartmental modeling of (1)(3)C-lycopene absorption, isomerization, and distribution kinetics in healthy adults. The American Journal of Clinical Nutrition, 102, 1436-1449.

Moran, N. E., Clinton, S. K., \& Erdman, J. W., Jr. (2013). Differential bioavailability, clearance, and tissue distribution of the acyclic tomato carotenoids lycopene and phytoene in mongolian gerbils. Journal of Nutrition, 143, 1920-1926.

Moran, N. E., Erdman, J. W., Jr., \& Clinton, S. K. (2013). Complex interactions between dietary and genetic factors impact lycopene metabolism and distribution. Archives of Biochemistry and Biophysics, 539, 171-180.

Moussa, M., Landrier, J. F., Reboul, E., Ghiringhelli, O., Coméra, C., Collet, X., et al. (2008). Lycopene absorption in human intestinal cells and in mice involves scavenger receptor class B type I but not Niemann-Pick C1-like 1. Journal of Nutrition, 138, 1432-1436.

Mozaffarian, D., Appel, L. J., \& Van Horn, L. (2011). Components of a cardioprotective diet: New insights. Circulation, 123, 2870-2891.

Muller, L., Caris-Veyrat, C., Lowe, G., \& Bohm, V. (2016). Lycopene and its antioxidant role in the prevention of cardiovascular diseases-A critical review. Critical Reviews in Food Science and Nutrition, 56, 1868-1879.

Murphy, M. M., Barraj, L. M., Spungen, J. H., Herman, D. R., \& Randolph, R. K. (2014). Global assessment of select phytonutrient intakes by level of fruit and vegetable consumption. British Journal of Nutrition, 112, 1004-1018.

Osganian, S. K., Stampfer, M. J., Rimm, E., Spiegelman, D., Manson, J. E., \& Willett, W. C. (2003). Dietary carotenoids and risk of coronary artery disease in women. The American Journal of Clinical Nutrition, 77, 1390-1399.

Palozza, P., Catalano, A., Simone, R. E., Mele, M. C., \& Cittadini, A. (2012). Effect of lycopene and tomato products on cholesterol metabolism. Annals of Nutrition \& Metabolism, 61, 126-134.

Palozza, P., Simone, R., Catalano, A., Russo, M., \& Bohm, V. (2012). Lycopene modulation of molecular targets affected by smoking exposure. Current Cancer Drug Targets, 12 , 640-657.

Petyaev, I. M. (2016). Lycopene deficiency in ageing and cardiovascular disease. Oxidative Medicine and Cellular Longevit, 2016, 3218605
Reboldi, G., Gentile, G., Angeli, F., Ambrosio, G., Mancia, G., \& Verdecchia, P. (2011) Effects of intensive blood pressure reduction on myocardial infarction and stroke in diabetes: A meta-analysis in 73,913 patients. Journal of Hypertension, 29, 1253-1269.

Riccioni, G., D'Orazio, N., Palumbo, N., Bucciarelli, V., Ilio, E., Bazzano, L. A., et al. (2009). Relationship between plasma antioxidant concentrations and carotid intimamedia thickness: The Asymptomatic Carotid Atherosclerotic Disease In Manfredonia Study. European Journal of Preventive Cardiology, 16, 351-357.

Ried, K., \& Fakler, P. (2011). Protective effect of lycopene on serum cholesterol and blood pressure: Meta-analyses of intervention trials. Maturitas, 68, 299-310.

Rutz, J. K., Borges, C. D., Zambiazi, R. C., da Rosa, C. G., \& da Silva, M. M. (2016). Elaboration of microparticles of carotenoids from natural and synthetic sources for applications in food. Food Chemistry, 202, 324-333.

Sawardekar, S. B., Patel, T. C., \& Uchil, D. (2016). Comparative evaluation of antiplatelet effect of lycopene with aspirin and the effect of their combination on platelet aggregation: An in vitro study. Indian Journal of Pharmacology, 48, 26-31.

Schweiggert, R. M., Kopec, R. E., Villalobos-Gutierrez, M. G., Hogel, J., Quesada, S. Esquivel, P., et al. (2014). Carotenoids are more bioavailable from papaya than from tomato and carrot in humans: A randomised cross-over study. British Journal of Nutrition, 111, 490-498.

Sesso, H. D., Liu, S., Gaziano, J. M., \& Buring, J. E. (2003). Dietary lycopene, tomatobased food products and cardiovascular disease in women. Journal of Nutrition, 133, 2336-2341.

Shardell, M. D., Alley, D. E., Hicks, G. E., El-Kamary, S. S., Miller, R. R., Semba, R. D., et al. (2011). Low-serum carotenoid concentrations and carotenoid interactions predict mortality in US adults: The Third National Health and Nutrition Examination Survey. Nutrition Research, 31, 178-189.

Siervo, M., Lara, J., Ogbonmwan, I., \& Mathers, J. C. (2013). Inorganic nitrate and beetroot juice supplementation reduces blood pressure in adults: A systematic review and meta-analysis. Journal of Nutrition, 143, 818-826.

Song, B., Liu, K., Gao, Y., Zhao, L., Fang, H., Li, Y., ... Pei, L. (2017). Lycopene and risk of cardiovascular diseases: A meta-analysis of observational studies. Molecular Nutrition Food Research, 61. http://dx.doi.org/10.1002/mnfr.201601009.

Stahl, W., \& Sies, H. (2005). Bioactivity and protective effects of natural carotenoids. Biochimica et Biophysica Acta, 1740, 101-107.

Stahl, W., von Laar, J., Martin, H. D., Emmerich, T., \& Sies, H. (2000). Stimulation of gap junctional communication: Comparison of acyclo-retinoic acid and lycopene. Archives of Biochemistry and Biophysics, 373, 271-274.

Steinberg, D., \& Witztum, J. L. (2010). Oxidized low-density lipoprotein and atherosclerosis. Arteriosclerosis, Thrombosis, and Vascular Biology, 30, 2311-2316.

Tapiero, H., Townsend, D. M., \& Tew, K. D. (2004). The role of carotenoids in the prevention of human pathologies. Biomedicine \& Pharmacotherapy, 58, 100-110.

Tavani, A., Gallus, S., Negri, E., Parpinel, M., \& La Vecchia, C. (2006). Dietary intake of carotenoids and retinol and the risk of acute myocardial infarction in Italy. Free Radical Research, 40, 659-664.

Thies, F., Masson, L. F., Rudd, A., Vaughan, N., Tsang, C., Brittenden, J., et al. (2012) Effect of a tomato-rich diet on markers of cardiovascular disease risk in moderately overweight, disease-free, middle-aged adults: A randomized controlled trial. The American Journal of Clinical Nutrition, 95, 1013-1022.

Thies, F., Mills, L. M., Moir, S., \& Masson, L. F. (2017). Cardiovascular benefits of lycopene: Fantasy or reality? Proceedings of the Nutrition Society, 76, 122-129.

Tong, C., Peng, C., Wang, L., Zhang, L., Yang, X., Xu, P., et al. (2016). Intravenous administration of lycopene, a tomato extract, protects against myocardial ischemiareperfusion injury. Nutrients, 8, 138.

Uppala, P. T., Dissmore, T., Lau, B. H., Andacht, T., \& Rajaram, S. (2013). Selective inhibition of cell proliferation by lycopene in MCF-7 breast cancer cells in vitro: A proteomic analysis. Phytotherapy Research, 27, 595-601.

Wang, X. D. (2012). Lycopene metabolism and its biological significance. The American Journal of Clinical Nutrition, 96, 1214S-1222S.

Wang, X., Ouyang, Y., Liu, J., Zhu, M., Zhao, G., Bao, W., et al. (2014). Fruit and vegetable consumption and mortality from all causes, cardiovascular disease, and cancer: Systematic review and dose-response meta-analysis of prospective cohort studies. BMJ, 349, g4490.

Wolak, T., \& Paran, E. (2013). Can carotenoids attenuate vascular aging? Vascular Pharmacology, 59, 63-66.

Xu, Y., Arora, R. C., Hiebert, B. M., Lerner, B., Szwajcer, A., McDonald, K., et al. (2014). Non-invasive endothelial function testing and the risk of adverse outcomes: A systematic review and meta-analysis. European Heart Journal - Cardiovascular Imaging, $15,736-746$.

Yeo, H. Y., Kim, O. Y., Lim, H. H., Kim, J. Y., \& Lee, J. H. (2011). Association of serum lycopene and brachial-ankle pulse wave velocity with metabolic syndrome. Metabolism, 60, 537-543.

Zhang, Y., Liu, Y., \& Lv, Q. (2016). DFT study on the quenching mechanism of singlet oxygen by lycopene. RSC Advances, 6, 98498-98505.

Zou, J., Feng, D., Ling, W. H., \& Duan, R. D. (2013). Lycopene suppresses proinflammatory response in lipopolysaccharide-stimulated macrophages by inhibiting ROS-induced trafficking of TLR4 to lipid raft-like domains. The Journal of Nutritional Biochemistry, 24, 1117-1122.

Zubair, N., Kooperberg, C., Liu, J., Di, C., Peters, U., \& Neuhouser, M. L. (2015). Genetic variation predicts serum lycopene concentrations in a multiethnic population of postmenopausal women. Journal of Nutrition, 145, 187-192. 